\title{
Evaluación de la repetibilidad y reproducibilidad en la determinación de la demanda química de oxígeno en aguas naturales, residuales y residuales tratadas bajo la NMX-AA-030-SCFI-2001 Y PROY-NMX-AA- 030/2-SCFI-2010
}

\author{
Nidia Saavedra Villarreal ${ }^{1 *}$, Jorge L. Guzmán-Mar ${ }^{1}$, Deyanira Aguirre Flores ${ }^{1}$, Sandra Medina ${ }^{2}$, Nidia \\ Molina Recio ${ }^{2}$. \\ ${ }^{I}$ Universidad Autónoma de Nuevo León (UANL), Facultad de Ciencias Químicas, Pedro de Alba s/n, Cd. Universitaria, San Nicolás de \\ los Garza, Nuevo León, C.P. 64570. México. \\ ${ }^{2}$ Laboratorio de Servicios Clínicos y Toxicológicos S.A. de C.V. \\ ${ }^{*}$ E-mail: nidia.saavedrav@uanl.mx
}

\section{Resumen}

La evaluación de la repetibilidad y reproducibilidad en los laboratorios acreditados hoy en día es una práctica frecuente solicitada por la Entidad Mexicana de Acreditación (EMA) como parte de los requerimientos de la norma NMX-EC17025-IMNC-2006. La confiabilidad del resultado de una medición es el factor de mayor importancia para la toma de decisiones de los usuarios de este resultado. El propósito de este trabajo fue evaluar la repetibilidad y reproducibilidad de dos métodos que aplican la NMX-AA-030-SCFI-2001 que está vigente contra el PROY-NMX-AA-030/1-SCFI-2010, esto se realizó en un laboratorio acreditado ante la EMA. Con la finalidad de verificar que ambos métodos cumplan con el contenido de la demanda química de oxígeno (DQO) en concentraciones altas $(800 \mathrm{mg} / \mathrm{L})$ en aguas residuales de acuerdo a las normas antes mencionadas. Cuando en el laboratorio se aplican métodos diferentes a la normativa vigente es necesario realizar esta misma prueba para presentar su evaluación de repetibilidad y reproducibilidad con el objetivo de estimar la veracidad y precisión del método de medición establecido en la NMX-CH-5725-IMNC.

\section{Introducción}

Cuando ejecutamos el método de prueba en un laboratorio en el cual se van a usar diferentes métodos, equipos o varios analistas realizan el mismo análisis, el laboratorio se ve obligado a descubrir todas las variaciones potenciales que puedan ocurrir cuando se utilice este o estos métodos de prueba. Debido a que ahora tenemos diferentes métodos, con iguales condiciones de trabajo como los son equipos y condiciones ambientales, se habrán introducido todas las condiciones intermedias y otras más. De este modo, debemos esperar tener una variabilidad entre los resultados de los diferentes métodos. La medida de esta variación será debida a las lecturas tomadas entre el operario que ejecuta la prueba, esto se conoce como la desviación estándar de la reproducibilidad. Entonces, reducimos la jerga estadística. Lo mismo ocurre con $\mathrm{R}$, que es la desviación estándar de la reproducibilidad por 2.8. Con estos cálculos llegamos al intervalo de reproducibilidad, que se utiliza para comparar la diferencia entre un par de resultados de prueba reales que podemos observar de dos métodos. Generalmente sólo se necesita una pequeña cantidad de repeticiones para cada protocolo de prueba en cada laboratorio. $\mathrm{Al}$ promediar los resultados de cada método nos damos cuenta de cuál debería ser el comportamiento de un laboratorio típico. Por supuesto, todo esto depende de la cantidad de analistas participantes y del grado en que representen el mundo real de los laboratorios.

\section{Definiciones}

a. Repetibilidad-. Indica el grado de acuerdo entre resultados mutuamente independientes de un ensayo, obtenidos utilizando el mismo método, en idénticos materiales, en el mismo laboratorio, por el mismo operador, usando el mismo equipo y en un corto intervalo de tiempo.

b. Reproducibilidad.- Indica el grado de acuerdo entre resultados mutuamente independientes de un ensayo obtenidos con el mismo método, en idénticos materiales, en diferentes laboratorios, con diferentes operadores y utilizando distintos equipos.

c. El contraste t.- Considera la diferencia entre dos métodos, entonces esta diferencia se obtiene de una población con media $\square \square_{\mathrm{d}}=0$.

\section{Metodología}

Para la presente evaluación de la repetibilidad y reproducibilidad se tomo como referencia la NMX-EC17025-INMC-2006 [2] y la NMX-CH-5725-IMNC. [3]. Requisitos para un experimento de veracidad y precisión: 1. En el esquema utilizado en el método básico, se envían muestras de $q$ lotes de materiales, representando $q$ niveles diferentes de ensayo bajo condiciones de repetibilidad, para cada uno de los $q$ niveles. Este tipo de experimento se denomina de nivel uniforme balanceado.

2. La realización de las mediciones debe estar perfectamente organizada, según las siguientes instrucciones: 
a) Cualquier verificación preliminar del equipo debe realizarse tal como se especifique en el método normalizado;

b) Cada grupo de $\mathrm{n}$ mediciones pertenecientes a un nivel, debe realizarse bajo condiciones de repetibilidad; es decir, en un breve intervalo de tiempo, por el mismo operador, y sin calibración intermedia del aparato, a menos que esto sea parte integral de la realización de las mediciones.

c) Es esencial que cada grupo de $\mathrm{n}$ ensayos bajo condiciones de repetibilidad sea realizado de forma independiente, como si se tratara de $n$ ensayos sobre materiales diferentes. Como regla general, aunque el operador sepa que esta verificando materiales idénticos, debe hacerse hincapié en las instrucciones en que el propósito del experimento es determinar las diferencias que puedan observarse de los resultados, en un ensayo real. Si se sospecha que a pesar de esta advertencia, los resultados previos pueden influir sobre los resultados de los ensayos siguientes $\mathrm{y}$, por tanto sobre la varianza de repetibilidad, debe considerarse si es pertinente el uso de $n$ muestras separadas en cada uno de los $q$ niveles, codificados de forma que el operador no pueda identificar cuáles son las replicas de un nivel dado.

d) No es esencial que todos los $q$ grupos de $\mathrm{n}$ mediciones se realicen estrictamente en un corto intervalo de tiempo; grupos de mediciones diferentes pueden realizarse en diferentes fechas.

e) Las mediciones de todos los $q$ niveles deben realizarse por un único y mismo operador $\mathrm{y}$, además, las $\mathrm{n}$ mediciones para un nivel dado deben realizarse utilizando el mismo equipo de medición.

f) Si en el curso de las mediciones un operador no puede concluir todas ellas, otro operador puede completar el trabajo, siempre y cuando la sustitución no tenga lugar dentro de un grupo de $\mathrm{n}$ mediciones de un nivel, sino que ocurran únicamente entre dos de los $q$ grupos. Cualquier cambio debe ser notificado junto con los resultados.

g) Debe fijarse un tiempo límite dentro del cual todas las mediciones deben ser realizadas. Esto puede ser necesario para limitar el tiempo transcurrido entre el momento de la recepción de las muestras y el del comienzo de las mediciones:

h) Todas las muestras deben estar claramente etiquetadas con el nombre del experimento y la identificación de la muestra.

La metodología para calcular la repetibilidad y reproducibilidad en la determinación de la demanda química de oxígeno en aguas residuales según norma NMX-AA-030-SCFI-2001 [6] se describe a continuación:
3. Especificaciones del mesurando. La determinación de la demanda química de oxígeno en aguas residuales se realizó empleando un método que se basa en que una gran cantidad de compuestos orgánicos e inorgánicos son oxidados con una mezcla de ácido crómico y sulfúrico a ebullición. La muestra se coloca a reflujo en una disolución de ácido fuerte con un exceso conocido de dicromato de potasio $\left(\mathrm{K}_{2} \mathrm{Cr}_{2} \mathrm{O}_{7}\right)$.

Después de la digestión, el dicromato no reducido se mide por titulación o espectrofotométricamente para determinar la cantidad de dicromato consumido y calcular la materia oxidable en términos de oxígeno equivalente. Procedimiento de acuerdo a la NMX-AA-030-SCFI-2001 método a reflujo cerrado/ método espectrofotométrico precalentar a $150^{\circ} \mathrm{C}$ el digestor de DQO y se colocan en los tubos de reacción 1,5 $\mathrm{mL}$ de la disolución de digestión A o B ( A alta concentración, B baja concentración), tomar cuidadosamente $2,5 \mathrm{~mL}$ de muestra previamente homogeneizada dentro de los tubos de reacción. Cerrar inmediatamente para evitar que se escapen los vapores, asegurarse de que están herméticamente cerrados. Suavemente invertir los tubos varias veces destapando después de cada inversión para liberar la presión. Añadir cuidadosamente $3,5 \mathrm{~mL}$ de la disolución de digestión respectiva y colocar $2,5 \mathrm{~mL}$ de agua en un tubo para la determinación del blanco de reactivos.

Colocar todos los tubos en el digestor previamente calentado a $150{ }^{\circ} \mathrm{C}$ y mantener a reflujo por $2 \mathrm{~h}$. Retirar los tubos del digestor y dejar que los tubos se enfríen a temperatura ambiente, permitiendo que cualquier precipitado se sedimente. Medir la absorbancia en el espectrofotómetro, previamente calibrado o cuantificar por titulación.

Para aguas que contengan una DQO baja (en el rango de 5 a $75 \mathrm{mg} / \mathrm{L}$ ), utilizar la disolución de digestión B. Si el valor de la DQO determinado es más alto que $75 \mathrm{mg} / \mathrm{L}$ después de usar estos reactivos, reanalizar la muestra, utilizando la disolución A.

Procedimiento de acuerdo a la PROY-NMX-AA-030SCFI-2010. PROCEDIMIENTO HACH (tubos adquiridos en forma comercial).

Siempre que sea posible se recomienda adquirir tubos sellados DQO-TS listos para su uso. Esto minimiza el manejo de productos químicos tóxicos por personal del laboratorio. Los tubos de tipo comercial se pueden comprar para cubrir diferentes intervalos de análisis, (por ejemplo 50, 160, 1000 ó $1500 \mathrm{mg} / \mathrm{L}$ ).

El intervalo de concentración DQO-TS de tubos comerciales será especificado por el fabricante y no deberá excederse. Si esto llegara a ocurrir, la muestra deberá diluirse convenientemente dentro del intervalo de concentración especificado.

Verificar si la disolución en el tubo muestra alguna traza de color verde; si es así, rechace el tubo. Encender el bloque de calentamiento y precalentar a $150{ }^{\circ} \mathrm{C}$, quitar la tapa del tubo de digestión y agitar para homogenizar la muestra e inmediatamente pipetear $2 \mathrm{~mL}$ de la muestra en el tubo de digestión.

Para cualquier muestra que se prevé que tenga un valor de DQO-TS mayor a $1000 \mathrm{mg} / \mathrm{L}$, pipetear en el tubo de digestión $2 \mathrm{~mL}$ de una porción de la muestra diluida apropiadamente. 
Llevar a cabo una determinación de blanco utilizando agua con cada lote de análisis. Colocar la tapa firmemente y mezclar el contenido invirtiendo suavemente el tubo varias veces. Limpiar el exterior del tubo con un papel suave.

Colocar el tubo en el bloque de calentamiento. Refluir el contenido a $150{ }^{\circ} \mathrm{C}$ durante $2 \mathrm{~h} \pm 10 \mathrm{~min}$. Retirar los tubos del bloque de calentamiento y dejar enfriar a $60^{\circ} \mathrm{C}$ o menos. Mezclar el contenido invirtiendo cuidadosamente cada tubo varias veces mientras permanezcan calientes. Después, dejar enfriar los tubos a temperatura ambiente antes de medir la absorbancia.

Para ambos métodos:

Ajustar el espectrofotómetro con el blanco reactivo a cero de absorbancia. Medir la absorbancia a $593 \mathrm{~nm}$ en una celda de cuarzo de $1 \mathrm{~cm}$ de paso óptico para las muestras y estándares.

Registrar las lecturas de las absorbancias. Determinar los $\mathrm{mg} / \mathrm{L}$ de DQO presentes en la muestra directamente de la curva de calibración. (Ec. 1).

$$
\mathbf{Y}=\mathbf{m X}+\mathbf{b}
$$

Dónde:
$\mathrm{m}$ es la pendiente.
b es la ordenada al origen.
$Y$ es la absorbancia.
$\mathrm{X}$ son los mg/L DQO.

4. El modelo matemático del mensurando. La expresión matemática para el cálculo de la demanda química de oxígeno toma en cuenta la lectura de absorbancia de la muestra.

5. Para la evaluación de los resultados se considera una de las propiedades más importantes de un método analítico es que debería estar libre de errores sistemáticos. Esta propiedad de un método analítico se puede contrastar al aplicar el método a una muestra de ensayo estándar que contenga una cantidad conocida de analito. Para decidir si la diferencia entre la cantidad medida y la cantidad conocida se puede atribuir a estos errores aleatorios, se puede aplicar una prueba estadística denominada contraste de significación. Como su nombre indica, esta aproximación contrasta si son significativas las diferencias entre los dos resultados, o si se pueden justificar sólo por variaciones aleatorias. Para llevar a cabo un contraste de significancia se prueba la veracidad de una hipótesis denominada hipótesis nula, denominada Ho. El término nulo se emplea para indicar que no hay otra diferencia entre el valor observado y el conocido que la atribuible a la variación aleatoria. Cuanto más pequeña sea la probabilidad de que la diferencia observada ocurra por azar, menos probable será que la hipótesis nula sea verdadera. Normalmente la hipótesis nula se rechaza cuando la probabilidad de que dicha diferencia observada ocurra por azar es menor que 1 en 20 veces (es decir, 0.05 ó $5 \%$ ).

El contraste considera la razón de la varianzas muestrales.

$$
\text { Ec. } \sigma=\sqrt{\frac{1}{N-1} \sum_{i=1}^{N}\left(x_{i}-\bar{x}\right)^{2}}
$$

El nivel de significancia se indica escribiendo, por ejemplo, $\mathrm{P}$ (es decir, la probabilidad) $=0.05$, y proporciona la probabilidad de rechazar una hipótesis nula cuando esta es verdadera.

Para decidir si la diferencia entre x y $\square$ es significativa, es decir para contrastar Ho: la media de la población es igual a $\square \square \square$ se calcula el estadístico t:

$$
|\mathrm{t}|=(\mathrm{x}-\square) \sqrt{\mathrm{n}} / \mathrm{s}
$$

Donde:

$\mathrm{x}=$ media muestral

$\square=$ la media de la población

$\mathrm{s}=$ desviación estándar muestral

$\mathrm{n}=$ tamaño muestra.

$\mathrm{Si}|\mathrm{t}|$ es mayor que un valor crítico entonces se rechaza la hipótesis nula. El valor crítico de $\mathrm{t}$ para un nivel significativo concreto para un tamaño muestral de 21 y un nivel de significancia de 0.05 el valor crítico $t_{20}=2.09$, donde, el subíndice se utiliza para indicar el número de grados de libertad.

6. En esta evaluación se realizó la prueba de ambos métodos en un mismo día, obteniéndose 21 resultados, de cada método para calcular la desviación estándar y el valor crítico de $t$ para un contraste de dos colas $(p=0.05)$ para cada método, por la Ec. 3. Una vez que se han determinando los valores de la desviación estándar y el valor crítico de t para un contraste de dos colas $(p=0.05)$ para cada analista, el análisis de los resultados se realiza mediante las siguientes técnicas:

7. La etapa final es calcular el contraste $|t|$ para la comparación del valor critico en los resultados presentados por los métodos A y B, con un nivel de significación de 0.05 para un contraste de dos colas. El Método A PROY-NMX-AA-030-SCFI-2010 y el Método B NMX-AA-030-SCFI-2001.

Los valores mostrados en la Tabla 1 corresponden a los datos obtenidos por los métodos $\mathrm{A}$ y $\mathrm{B}$ durante su evaluación de repetibilidad y reproducibilidad de resultados de la determinación del contenido de la demanda química de oxígeno en aguas residuales de acuerdo a la NMX-AA-030-SCFI-2001. 
Tabla 1. Resultados de la medición de la demanda química de oxígeno por dos métodos

\begin{tabular}{|c|c|c|c|}
\hline \multicolumn{4}{|c|}{ DQO } \\
\hline & $\begin{array}{c}\text { PROY-NMX- } \\
\text { AA-030/1- } \\
\text { SCFI-2010 } \\
\end{array}$ & $\begin{array}{c}\text { NMX-AA- } \\
\text { 030-SCFI- } \\
2001 \\
\end{array}$ & DIFERENCIA \\
\hline 1 & 92.78 & 93.46 & 0.7 \\
\hline 2 & 102.85 & 93.46 & -9.4 \\
\hline 3 & 122.99 & 93.46 & -29.5 \\
\hline 4 & 102.85 & 93.46 & -9.4 \\
\hline 5 & 95.30 & 91.52 & -3.8 \\
\hline 6 & 92.78 & 93.46 & 0.7 \\
\hline 7 & 85.23 & 91.52 & 6.3 \\
\hline 8 & 77.67 & 89.58 & 11.9 \\
\hline 9 & 75.16 & 91.52 & 16.4 \\
\hline 10 & 75.16 & 91.52 & 16.4 \\
\hline 11 & 80.19 & 93.46 & 13.3 \\
\hline 12 & 75.16 & 91.52 & 16.4 \\
\hline 13 & 82.71 & 89.58 & 6.9 \\
\hline 14 & 82.71 & 89.58 & 6.9 \\
\hline 15 & 100.33 & 93.46 & -6.9 \\
\hline 16 & 82.71 & 93.46 & 10.8 \\
\hline 17 & 102.85 & 97.33 & -5.5 \\
\hline 18 & 87.74 & 97.33 & 9.6 \\
\hline 19 & 95.3 & 95.39 & 0.1 \\
\hline 20 & 85.23 & 97.33 & 12.1 \\
\hline 21 & 92.78 & 93.46 & 0.7 \\
\hline $\mathrm{X}$ & 90.02 & 93.09 & 3.07 \\
\hline $\mathrm{S}$ & 12.0 & 2.3 & 11.3 \\
\hline $\mathrm{CV}$ & 13.4 & 2.5 & \\
\hline & & Error std & 2.46 \\
\hline & & $\mathrm{T}$ & 1.24 \\
\hline & & $\mathrm{T}_{95 \%}=$ & 2.09 \\
\hline
\end{tabular}

\section{Resultados y discusión}

La evaluación de los resultados de la determinación del contenido de la demanda química de oxígeno de acuerdo a la NMX-AA-030-SCFI-2001 realizada en este trabajo se llevó a cabo en aguas residuales en una concentración baja de $100 \mathrm{mg} / \mathrm{L}$. Evaluamos el contraste t, para decidir si la diferencia entre x y $\mu$ es significativa, si $|t|$ es mayor que un cierto valor crítico entonces se rechaza la hipótesis nula, en donde se supuso que los resultados del contenido de la demanda química de oxígeno de los métodos A y B no diferían significativamente.
Este supuesto se puede contrastar ahora. La t 1.24 pasa con un nivel de confianza de $95 \%$. Calculado t:

$$
\mathrm{t}=\frac{(\mathrm{x}-\mu) \sqrt{\mathrm{n}}}{\delta}
$$

El valor critico encontrado para $t_{20}=2.09$ con un nivel de confianza de $95 \%$, el valor calculado es menor que este por tanto no hay diferencia significativa entre $\mathrm{x}$ y $\mu$ a un nivel del 0.05 , estos valores nos indican que tenemos una reproducibilidad y repetibilidad de resultados confiables entre los métodos A y B, ya que estos se encuentra dentro de un grado de confianza del $95 \%$, además demuestra que el proceso de la prueba de contenido de la demanda química de oxígeno de acuerdo a la NMX-AA030-SCFI-2001 y PROY-NMX-AA-030/1-SCFI-2010 tiene un alto grado de confiabilidad en los valores obtenidos en este laboratorio.

\section{Conclusiones}

Se concluye que esta evaluación de contraste de significación de resultados entre dos métodos que se compara su precisión para la misma prueba proporciona una idea clara del nivel de confianza al ejecutar dicha prueba, el cual cumple satisfactoriamente, se presentaron resultados en los cuales se observa que el valor critico es menor al valor calculado $|t|$ de los resultados del contenido de la demanda química de oxígeno de los métodos A y B, con un nivel de confianza de un $95 \%$.

\section{Referencias}

1.Miller J.N., Miller J.C. Estadística y Quimiometría para Química Analítica, 4a Edición, Editorial Prentice Hall (2004).

2.NMX-17025-IMNC-2006. IMNC. Requisitos generales para la competencia de los laboratorios de ensayo y de calibración. Instituto Mexicano de Normalización y Certificación, A.C. (2006).

3.NMX-CH-5725-1- IMNC- 2006. Exactitud (veracidad $\mathrm{y}$ precisión) de resultados y métodos- parte 1: Principios generales y definiciones.

4.NMX-CH-5725-2-IMNC-2006. Exactitud (veracidad y precisión) de resultados y métodos de medición - Parte 2: Método básico para la determinación de la repetibilidad y la reproducibilidad de un método de medición normalizado.

5.NMX-CH-5725-6-IMNC-2006. Exactitud (veracidad y precisión) de resultados y métodos de medición - Parte 6: Utilización en la práctica de los valores de exactitud.

6.NMX-AA-030-SCFI-2001 Análisis de aguadeterminación de la demanda química de oxígeno en aguas naturales, residuales y residuales tratadas.

7.PROY-NMX-AA-030/2-SCFI-2010 Análisis de aguadeterminación de la demanda química de oxígeno en aguas naturales, residuales y residuales tratadas. 